\title{
Transformation of Old Concepts for a New Era of Cancer Immunotherapy: Cytokine Therapy and Cancer Vaccines as Combination Partners of PD1/PD-L1 Inhibitors
}

\author{
Romualdo Barroso-Sousa ${ }^{1}$ - Patrick A. Ott ${ }^{1}$ \\ Published online: 15 November 2018 \\ (C) Springer Science+Business Media, LLC, part of Springer Nature 2018
}

\begin{abstract}
Purpose of Review Immune checkpoint inhibitors (ICI) are only effective in a subset of patients. Here, we will review the rationale and data supporting the combination of PD-1 pathway inhibition with recombinant cytokines and neoantigen-based cancer vaccines that can potentially increase the number of patients who will benefit from immunotherapy.

Recent Findings The safety and tolerability of new interleukin(IL)-2 formulations, IL-15 super agonist, and PEGylated IL-10 have been evaluated in early phase clinical trials with promising efficacy data, both as monotherapy and in combination with ICI. Larger studies focusing on the efficacy of these combinations are ongoing. Personalized neoantigen-based cancer vaccines, enabled by improvements in sequencing computational capabilities, have been proven to be feasible, safe, and able to trigger a consistent vaccine-specific immune response in cancer patients.

Summary New pharmacologically modified recombinant cytokines and personalized neoantigen-based vaccines may turn these approaches into powerful tools for effective combination immunotherapy.
\end{abstract}

Keywords Atezolizumab - Immune checkpoint inhibitors · Interleukin-2 · Interferon · Interleukin 10 - Interleukin 15 . Ipilimumab $\cdot$ NKTR-214 $\cdot$ Neoantigens $\cdot$ Nivolumab $\cdot$ PD-1 inhibitors $\cdot$ PD-11 inhibitors $\cdot$ Pembrolizumab $\cdot$ Vaccines

\section{Introduction}

Immune checkpoint inhibitors (ICI) are now considered one of the pillars of anticancer therapy. In particular, anti-PD-1/ PD-L1 antibodies have demonstrated broad clinical efficacy, and currently, these agents are approved for the treatment of several different cancer types, including one histologyagnostic approval for patients with unresectable or metastatic, microsatellite instability-high (MSI-H) or mismatch repairdeficient tumors [1-3]. However, despite these encouraging results, the majority of patients do not derive benefit from ICI when they are given in monotherapy, suggesting that there must be additional factors causing resistance to ICI. Thus, it is likely that combinatorial approaches will be needed, i.e.,

This article is part of the Topical Collection on Melanoma

Patrick A. Ott

patrick_ott@dfci.harvard.edu

1 Department of Medical Oncology, Dana-Farber Cancer Institute, 450 Brookline Avenue, Dana 2127, Boston, MA 02215, USA adding other agents to anti-PD-1/PD-L1 therapy, in order to increase the number of patients who respond in a given tumor type, have complete, and most importantly, have durable responses.

Towards this end, the combination of the anti-CTLA-4 antibody ipilimumab and the anti-PD-1 antibody nivolumab has been shown superiority over ipilimumab alone in advanced melanoma $[4,5]$. However, this regimen is associated with high $(>50 \%)$ rates of severe immune-related adverse events (irAEs), including dermatologic, endocrine, gastrointestinal, pulmonary, and hepatic toxicities [4]. Moreover, a still significant proportion of patients showed primary resistance to this therapy, indicating a need for alternative strategies for these patients. The broad efficacy of ICI across cancers supports the rationale for further development of anticancer immunotherapy in general and provides a rationale to revisit "older" immunotherapy approaches. Importantly, many of these approaches rely on the activation of the first steps of the cancer-immunity cycle [6] (antigen presentation, activation of APCs, and activation and survival of naïve $T$ cells) and can synergize with ICI, which works by unleashing $\mathrm{T}$ cells from negative regulatory mode. In this review, we will review cytokine therapy and cancer 
vaccines as potential combination partners with PD-1/PD-L1 inhibitors for the treatment of melanoma and other cancers.

\section{Interleukin (IL)-2}

\section{High-Dose IL-2}

The recognition that a cytokine now called IL- 2 could expand $\mathrm{T}$ cells and sustain their functional activity was crucial for the current understanding of the cellular aspects of anticancer immunity as well as the development of cancer immunotherapy [7]. Interleukin-2 is secreted predominantly by activated CD4 $\mathrm{T}$ cells, but it can also be released by other immune cells, including CD8 T cells, natural killer (NK) cells, and dendritic cells (DCs). Following its binding to the receptor-signaling complex containing alpha (IL2R $\alpha, \mathrm{CD} 25$ ), beta (IL2R $\beta$, $\mathrm{CD} 122$ ), and common gamma chain receptors (IL2R $\gamma$, CD132) [8], IL-2 acts as a potent T cell growth factor, with a pleiotropic effect on both CD8 and CD4 T cell function, survival, and differentiation [9]. At high doses, IL-2 binds to heterodimeric IL2R $\beta \gamma$ causing expansion of CD8 T cells [10]. IL-2 also binds with higher affinity to its heterotrimeric receptor containing the subunit IL-2R $\alpha$ (CD25) forming the IL- $2 \alpha \beta \gamma$ complex, leading to expansion and maintenance of $\mathrm{T}$ regulatory (T-reg) cells [7], which constitutively express high levels of IL-2R $\alpha$.

Pioneering work by Rosenberg [11] and colleagues demonstrated that administration of recombinant IL-2 led to substantial regression of established tumor metastases in murine models, paving the way to the first clinical trials using recombinant IL-2 in patients with advanced cancer. The studies demonstrated for the first time that targeted immune intervention with stimulated $\mathrm{T}$ lymphocytes could mediate complete elimination of metastatic cancers in humans. However, IL-2 is effective only in a small fraction of patients with advanced melanoma or renal cell cancer and requires administration in high doses, resulting in substantial toxicity related to the cytokine outburst, with a reported treatment-related mortality rate of 2-4\% [12-16]. Retrospective series of patients with advanced melanoma and renal cell carcinoma (RCC) showed that the objective response achieved with the approved regimen of high-dose IL-2 is between 15 and $20 \%$ in these patients [12-16]. Importantly, approximately $5 \%$ of patients achieved durable response, some of which have lasted for 20 years or longer. This excellent response durability associated with high-dose IL-2 played a role in the Food and Drug Administration (FDA) approval of recombinant IL-2 for the treatment of advanced renal cell carcinoma in 1992 and metastatic melanoma in 1998.

A more contemporary experience, including 170 patients with melanoma and 192 patients with RCC treated with highdose IL-2 between 2005 and 2012 from 40 centers, showed similar objective response rates, with longer overall survival than historical control [17]. However, because of the serious AEs that include hypotension, cardiac arrhythmias, metabolic acidosis, dyspnea, and renal failure, neurotoxicity, and dermatologic complications, the administration of high-dose IL-2 requires an in-patient setting where close monitoring and blood pressure support can be provided. Although these toxicities are manageable and reversible after stopping IL-2 infusion, they limit the use of IL-2 outside large and experienced centers. This limitation, in conjunction with the low response rates and the emergence of ICI, led to a decrease in the utilization of high-dose IL-2.

\section{NKTR-214}

NKTR-214 is a conjugated IL-2 and itself conjugated to 6 releasable polyethylene glycol (PEG) chains. PEGylation of recombinant molecules can improve half-life and biological activity [18] and, depending on where the PEG chains are located, can also alter its target cell specificity. In the case of NKTR-214, PEG chains are slowly released leading to generation of active IL-2 conjugates. In addition, PEG chains are strategically located to mask the region of IL-2 that interacts with the IL2R $\alpha$ subunit responsible for activating T-regs, with no impairment at the binding site to IL2R $\beta$. In preclinical studies, NKTR-214 was shown to increase the ratio of CD8/ T-reg cells in the tumor microenvironment and mediated antitumor activity in aggressive murine tumors as a single agent and in combination with anti-CTLA-4. Furthermore, the molecule was well tolerated in non-human primates, without evidence of hypotension or vascular leak syndrome at its maximum tolerated dose.

The phase 1 dose escalation trial of NKTR monotherapy assessing safety and tolerability enrolled 25 patients with locally recurrent or metastatic solid tumors including RCC $(N=$ 16), melanoma $(N=6)$, and other solid tumors [19] (NCT02869295). Patients received outpatient intravenous dosing over 15 min every 2 or 3 weeks. NKTR-214 had a favorable safety profile, with no immune-related AEs; grade 3 hypotension was observed in $3 / 25$ patients and was rapidly reversible with fluids; notably, all three patients continued dosing after these events; no capillary leak syndrome was observed. Furthermore, the drug showed antitumor activity, with antitumor immune response in agreement with preclinical data: elevation of newly proliferating Ki67+ CD8+ $\mathrm{T}$ cells and NK cells in the blood also observed in the tumor; greater abundance of $\mathrm{CD} 8+\mathrm{T}$ cells to $\mathrm{T}$ regulatory immune suppressive cells accumulating in the tumor and increased PD-1 expression on CD4+ and CD8+ T cells in blood and tumor. Clinical trials evaluating the combination of NKTR-214 and PD-1/PD-L1 inhibitors are ongoing and will be discussed below. 


\section{Combination of IL-2 and PD-1/PD-L1 Inhibitors}

Even if better tolerated formulations of IL-2 will be developed, the low overall response rate is still the problem regarding most patients who do not benefit from monotherapy. At the time of the clinical development of the regimen with highdose IL-2 in patients with advanced cancer, little was known about the mechanisms regulating $\mathrm{T}$ cell activation. The discovery and investigation of CTLA-4 and subsequently PD-1 and its ligands PD-L1 and PD-L2 in the context of cancer therapy brought to light the critical role of these immune checkpoints in the anti-tumor immune response and provided the basis for the clinical development of ICI [20-24].

Based on their distinct roles of IL-2 and PD-1-PD-L1 interactions within the cancer immunity cycle [6], IL-2 and PD-1/ PD-L1 blockade have the potential to act synergistically. While IL-2 secretion is relevant during the priming and activation of tumor-specific T cells, PD-1/PD-L1 inhibitors act "downstream" during the effector phase in the tumor. In line with this hypothesis, West et al. showed that PD-L1 blockade synergizes with IL-2 therapy in enhancing virus-specific CD8+ T cell responses and decreasing viral load in a mouse model of chronic lymphocytic choriomeningitis virus (LCMV) infection [25].

Preliminary results of the phase $1 / 2$ PIVOT- 02 trial (NCT02983045), evaluating the safety and activity of the combination of NKTR-214 and Nivolumab in patients with advanced melanoma, RCC, non-small cell lung cancer (NSCLC) were recently presented [26•]. Following the doseescalation phase of the study, the recommended phase 2 dose was the combination of NKTR-214 $0.006 \mathrm{mg} / \mathrm{kg} \mathrm{q3w}+$ nivolumab $360 \mathrm{mg} \mathrm{q} 3 \mathrm{w}$. The phase 2 portion of the trial is ongoing and is planned to accrue a total of 330 patients with different cancer types, including advanced melanoma, NSCLC, RCC, bladder cancer, and triple-negative breast cancer. At ASCO 2018 Diab et al. presented data for frontline melanoma, frontline RCC, and frontline cisplatin-ineligible urothelial carcinoma (UC) cohorts included in the phase 2 portion of the trial. As of May 2018, the best overall response by RECIST 1.1 was $50 \%(14 / 28)$ in treatment-naive melanoma patients; 46\% (12/26) in treatment-naive RCC patients; $14 \%(6 / 10)$ in treatment-naive cisplatin-ineligible UC patients. Safety data were available for 283 patients treated at the RP2D. Grade $\geq 3$ treatment-related AEs occurred in $14.1 \%$ of patients, including syncope $(n=5)$, hypotension $(n=5)$, rash $(n=4)$, increased lipase $(n=4)$, and dehydration $(n=3)$. Grade $\geq 3$ irAEs were reported in $3.5 \%$ of patients. One patient died of grade 5 pneumonitis. Another phase 1 study (NCT03138889) is evaluating the safety of the combination of NKTR-214 with Pembrolizumab or with Atezolizumab in patients advanced NSCLC, bladder cancer, and melanoma. Currently, multiple clinical trials are ongoing evaluating the combination of high- and low-dose recombinant IL-2 with PD-1/PD-L1 inhibitors.

\section{Interleukin 15}

Both IL-2 and IL-15 signal through the IL-2R $\beta \gamma$ heterodimeric receptor. Therefore, similar to IL-2, IL-15 promotes CD8-positive $\mathrm{T}$ cell and NK cell activation and proliferation. However, IL-2 also leads to expansion of T-reg cells that express the unique IL- $2 \mathrm{R} \alpha$ subunit, facilitating high-affinity IL-2 binding. Preclinical studies in mice suggested that IL-15 had a more favorable toxicity profile than IL-2, with robust efficacy, including induction of antitumor immunity [27-29], leading to the possibility that IL-15 could in fact be a therapeutic alternative to IL-2 [10]. Treatment with recombinanthuman IL-15 (rhIL-15) was shown to be safely administered to patients and to induce biological activity on NK cells and CD8+ memory T cells [30]. While promising antitumor activity of IL-15 was seen preclinically, it was observed that its biological activity could be further augmented by pre-association with its soluble receptor (IL-15R $\alpha)$ [31-35].

ALT-803 is a pharmacological grade IL-15/IL- $15 \mathrm{R} \alpha$ complex fused to an IgG1 Fc, in which IL-15 is additionally mutated (asn72asp) to further increase biological activity making this complex an IL-2 and IL-15R $\beta \gamma$ superagonist [36]. Recently, Wrangle et al. reported the results of a phase $1 \mathrm{~b}$ trial assessing the safety of ALT-803 in combination with nivolumab and the anti-tumor activity of the combination in patients with advanced NSCLC [37०]. The study enrolled patients with previously treated stage IIIB or IV NSCLC from three academic hospitals in the United States. Patients received the IL-15 superagonist ALT-803 subcutaneously once per week, on weeks 1 to 5 of four 6-week cycles for 6 months in escalating dose combination with nivolumab. The primary endpoint was to define safety and tolerability and to establish a recommended phase 2 dose of ALT-803 in combination with nivolumab. A total of 23 patients were enrolled and 21 were treated at four dose levels of ALT-803 in combination with nivolumab. No dose-limiting toxicities were observed, and the maximum tolerated dose was not reached. The most common adverse events were injection-site reactions (90\% of patients) and flu-like symptoms (71\%). The most common grade 3 adverse events, occurring in two patients each, were lymphocytopenia and fatigue. No grade 4 or 5 adverse events were recorded. The recommended phase 2 dose of ALT- 803 is $20 \mu \mathrm{g} / \mathrm{kg}$ given once per week subcutaneously in combination with $240 \mathrm{mg}$ intravenous nivolumab every 2 weeks, and the ongoing portion of this trial is ongoing. In a post hoc analysis, six $(29 \%)$ of 21 patients achieved an objective response classified as partial response per RECIST 1.1. Notably, 11 patients had previously received single-agent PD-1 monoclonal antibody and progressed after at least 3 months of treatment, and three $(27 \%)$ of them achieved partial response and seven (64\%) stable disease. 


\section{Interferon-Alpha}

For two decades, high-dose interferon- $\alpha$ (IFN- $\alpha$ ) was the only approved adjuvant therapy for the treatment of stage III highrisk melanoma; however, the drug was not consistently used mainly because of considerable toxicity and conflicting data regarding overall survival benefit. Efficacy was initially demonstrated in the Eastern Cooperative Oncology Group (ECOG) 1684 [38] and Intergroup E1694 studies [39], as well as a meta-analysis that included results from trials with different regimens of IFN- $\alpha$ [40]. PD-1 inhibition (in addition to targeted therapy for BRAFV600 mutant melanoma) now has largely replaced IFN- $\alpha$ for the treatment of resected stage III melanoma [41, 42]. IFN- $\alpha$ is also approved as therapy for patients with advanced RCC, in combination with bevacizumab [43]. With regards to the potential of IFN- $\alpha$ as a combination partner with PD-1/PD-L1 inhibitors, preclinical data showed that IFN- $\alpha$ increases and prolongs PD-1 expression on T cells, and the combination of this cytokine with PD1 inhibition was synergistic [44]. Thus, there was a compelling rationale to explore IFN- $\alpha$ in combination with PD-1 inhibition. Nevertheless, only modest clinical efficacy was seen in patients who were treated with maximum tolerated doses of pembrolizumab plus PEGylated IFN- $\alpha$ on the phase $1 b$ trial KEYNOTE-029 (NCT02089685) [45].

\section{IL-10}

IL-10 is produced by activated T cells and antigen presenting cells; its cognate receptor IL-10R is induced in $\mathrm{CD} 8^{+} \mathrm{T}$ cells upon antigen recognition. Traditionally, this cytokine has been considered as anti-inflammatory, and recombinant IL-10 has been studied in clinical trials as an anti-inflammatory molecule in psoriasis, inflammatory disease, and liver fibrosis [46]. Altogether, it was supposed that IL-10 would negatively impact anticancer immunity. However, at higher doses, IL-10 was shown to cause activation and proliferation of intratumoral CD8+ T cells [47, 48]. To allow sustained high systemic concentrations of IL-10, a pegylated recombinant IL-10 called AM0010 was developed. Preclinical data showed that this form of IL-10 leads to the expansion, activation, and cytotoxicity of tumor-infiltrating CD8+ T cells [49]. In preclinical tumor models, AM0010 induces a CD8+ T cell-mediated rejection of large tumors and metastases [49].

A phase $1 / 1 \mathrm{~b}$ basket trial is evaluating the safety, pharmacodynamics, and antitumor activity of AM0010 monotherapy or in combination with chemotherapy or PD-1 inhibitors (NCT02009449). Preliminary results on 33 heavily pretreated cancer patients treated with daily subcutaneous injection of AM0010 in the dose escalation phase of this trial showed that the drug is well tolerated, and a maximum tolerated dose was not defined [50]. The most frequently observed AEs were anemia (51\%), fatigue (45\%), thrombocytopenia (42\%), injection site reactions $(36 \%)$, and fever $(30 \%)$. Grade 3 to 4 nonhematopoietic treatment-related AEs were observed in $15 \%$ of patients. Treatment-related grade 3 to 4 anemia or thrombocytopenia was observed in six patients (18\%). The RP2D was identified as $20 \mathrm{mg} / \mathrm{kg}$. An additional expansion cohort including 18 patients with RCC received AM0010 at a dose of $20 \mathrm{mg} / \mathrm{kg}$. The type and frequency of AEs observed in the expansion cohort in patients with RCC were comparable in number and severity to those observed at the $20-\mathrm{mg} / \mathrm{kg}$ dose in the escalation cohort. Among the 15 patients who had at least one scheduled assessment during treatment, four had a PR (27\%). More recently, safety and efficacy results of the cohorts combining AM00100 (10 mg/kg or $20 \mathrm{mg} / \mathrm{kg})$ with PD-1 inhibitors (pembrolizumab or nivolumab) were presented [51•]. The combination was well tolerated with no significant increase in $\mathrm{AE}$ profile over either agent in monotherapy. Furthermore, the combination weer suggestive of synergy with ORR of $41 \%$ ( 15 of 34 ) and $41 \%$ (11 of 27 ), respectively, in patients with RCC and NSCLC. Correlative studies demonstrated clonal T cell expansion upon treatment with AM0010 alone as well as the combination of AM0010 and PD-1 inhibition.

\section{Vaccines}

The efficacy of most cancer immunotherapy approaches critically depends on tumor-specific effector T cells that can traffic to the tumor microenvironment and mediate specific lysis of cancer cells. While many immunotherapies rely on endogenously activated tumor-specific $\mathrm{T}$ cells, cancer vaccines are aimed at inducing de novo tumor-specific $\mathrm{T}$ cell responses, as well as amplifying and expanding the antigen repertoire of pre-existing tumor-directed $\mathrm{T}$ cell responses. The successful engagement of professional antigen-presenting cells (APCs), most importantly DCs, is key to the efficacy of any cancer vaccine. Antigen presenting cells process tumor antigens and present antigen fragments (peptides) in the context of major histocompatibility complex (MHC) molecules to T cells in the draining lymph nodes, leading to activation and priming of $\mathrm{T}$ cells specific for these antigens.

To generate an effective cancer vaccine, four components must be addressed: (1) selection of a suitable tumor antigen as well as (2) immune-adjuvant, (3) formulation, and (4) vehicle used to deliver the antigenic target (Table 1). The different types of immune adjuvants, formulations, and delivery methods have been reviewed elsewhere [52]. Tumor antigens can be categorized as tumor-associated antigens (TAA) or tumor-specific antigens (TSA). Tumor-associated antigens are proteins that are (i) overexpressed (such as human epidermal growth factor 2, HER2; human telomerase reverse transcriptase, TERT), (ii) involved in tissue differentiation (such as mammaglobin-A; prostate-specific antigen, PSA), or the so 
Table 1 Key ingredients of a cancer vaccine

\begin{tabular}{|c|c|c|}
\hline Antigens: & Formulation/delivery: & Immune adjuvants: \\
\hline \multirow[t]{2}{*}{$\begin{array}{l}\text { - Cancer testis antigens } \\
\text { (e.g., NY-ESO-1) }\end{array}$} & $\begin{array}{l}\text { - Protein } \\
\text { - Peptide }\end{array}$ & $\begin{array}{l}\text { - TLR agonists (e.g., poly-ICLC, } \\
\text { imiquimod, CPG) }\end{array}$ \\
\hline & $\cdot$ DNA & - Cytokines (e.g., GM-CSF) \\
\hline \multirow{2}{*}{$\begin{array}{l}\text { - Differentiation antigens } \\
\text { (e.g., MART-1 etc) }\end{array}$} & - RNA & - STING activation \\
\hline & $\begin{array}{l}\text { - Autologous tumor cells } \\
\text { - Dendritic Cells }\end{array}$ & $\begin{array}{l}\text { - Saponin-based agents (e.g., ISCOMATRIX } \\
\text { - Agonistic antibodies (e.g., CD40, DEC205) }\end{array}$ \\
\hline Overexpressed antigens: & - Viral/bacterial vector & \\
\hline • (e.g., Her-2 neu) & 1 & • Emulsions (Montanide) \\
\hline • Viral antigens (e.g., HPV) & & • Liposomes \\
\hline $\begin{array}{l}\text { - Neoantigens (based on tumor } \\
\text { mutations) }\end{array}$ & & • Nanodiscs \\
\hline
\end{tabular}

$C p G O D N$ CpG oligodeoxynucleotide, GM-CSF granulocyte-macrophage colony-stimulating factor, poly-ICLC polyinosinic-polycytidylic acid with polylysine and carboxymethylcellulose, STING stimulator of interferon gene protein, TCR T cell receptor, TLR toll-like receptor called cancer-testis antigen, that are preferentially expressed by tumor cells (and germline and trophoblastic cells) instead of normal tissues (such as melanoma-associated antigen, MAGE; cancer-testis antigen 1,NY-ESO-1), except for fetal or immune privileged tissues). Tumor-specific antigens derived from an oncogenic virus can be found in hepatitis B virus-associated hepatocellular carcinoma, human papillomavirus (HPV)-associated cervical and head and neck cancers, and the Merkel cell polyomavirus-associated Merkel cell carcinoma. Since oncogenic viruses are associated with only a relatively small subset of cancers, these antigens are not suitable for broad use in cancer vaccines. In contrast, neoantigens which arise from somatic mutations, most of which are personal, i.e., not recurrent across a specific tumor type, are broadly expressed across the spectrum of solid and hematologic malignancies. In contrast to TAAs, neoantigens are not affected by central tolerance and are exquisitely specific to tumors (i.e., not expressed in healthy tissues). As a result, neoantigens have been established as highly suitable for use in cancer vaccines. Several lines of evidence support this notion: (1) higher neoantigen load has been associated with better clinical outcome in cancer patients both prognostically and in the context of immune checkpoint blockade [53-60]; (2) neoantigen-specific $\mathrm{T}$ cell populations are expanded in settings of effective antitumor immunity following the use of ICI [57, 58, 61]; (3) preclinical and human studies have demonstrated that neoantigen-specific $\mathrm{T}$ cells are cytolytic for tumor cells that present mutated peptides, and they can contribute to tumor regression [62-69].

Despite a relatively long history of cancer vaccine development, clinical efficacy has so far been limited; even the only FDA-approved cancer vaccine Sipuleucel-T only provides a 4-month overall survival benefit for patients with hormoneresistant prostate cancer with limited disease burden [70]. However, technological advances including fast and relatively cost-efficient next generation sequencing methods now enable the utilization of personal neoantigens; moreover, innovations in adjuvant technology and delivery methods are on the horizon [71].

\section{Personalized Cancer Vaccines Targeting Tumor Neoantigens}

Taking into consideration the genetic heterogeneity of tumor cells (both between individuals with the same type of cancer and even within individual tumors), and the complexity and diversity of HLA molecules, a critical challenge for personalized vaccines is the fact that the spectrum of neoepitopes derived from the same neoantigen may be very diverse across individuals. Furthermore, past studies have suggested that vaccines targeting single tumor antigens are unlikely to be successful due to tumor heterogeneity, and cancer immune editing, ultimately leading to clonal selection and immune evasion by the cancer cells [72••]. Consequently, targeting multiple neoantigens is likely required for personalized cancer vaccines to be effective.

To achieve this goal, it is necessary to accurately determine the cancer mutanome (the mapping of all mutations in a cancer) in order to select the most immunogenic neoantigens for use in the vaccine Fig. 1, an effort that is now feasible in the clinical arena largely due to the rapid evolution of sequencing and bioinformatic tools. Tumor DNA and RNA need to be extracted from single-cell suspensions of cells and matched normal tissue cells. Tumor somatic mutations are identified by whole-exome sequencing (WES) and orthogonally validated by RNA sequencing (to confirm mutations that likely result in altered gene expression). HLA typing should be carried out, in order to predict the affinity of the neoepitopes for binding the specific HLA type of that individual, thereby generating candidate vaccine epitopes. Validated epitopes are selected for incorporation into the personalized cancer vaccine, which is 


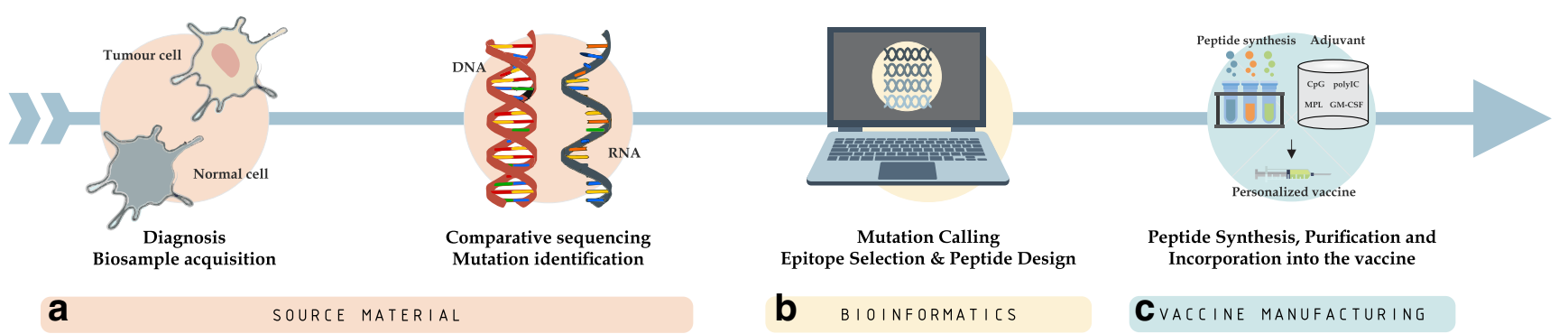

Figure 1 Workflow of manufacturing a personalized neoantigen-based cancer vaccine. a Source material and next-generating sequencing: Patient tumor biopsies and matched normal cells (e.g., peripheral blood white blood cells) are obtained and undergo next-generation sequencing; Tumor DNA is compared with germ line DNA, and tumor-specific nonsynonymous single-nucleotide variations or short indels in proteincoding genes are identified. RNA sequencing (RNA-seq) is used to orthogonally validate mutations of expressed genes. b Bioinformatics

administered to patients in combination with an immune adjuvant.

Recently, we and two other groups reported feasibility, safety, and immunogenicity of neoantigen-based cancer vaccines tested in three independent cohorts and phase 1

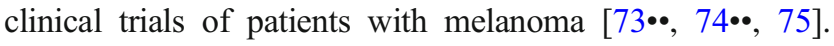
Different vaccine platforms were used in these three reports. The first included three patients with advanced melanoma previously treated with ipilimumab. In this study, autologous DCs were pulsed ex vivo with seven synthetic (8-10-mer) HLA-A2-restricted neoantigen peptides and injected intravenously [73••]. Both de novo and expansion of pre-exisiting neoantigen $\mathrm{CD} 8+\mathrm{T}$ cell responses were observed and vaccination led to an expansion of the $\mathrm{T}$ cell receptor repertoire. Our group conducted a study in patients with high-risk melanoma using a peptide vaccine (NeoVax) based on the neoantigen discovery pipeline. Synthetic long peptides (15-30-mers) representing up to 20 neoantigens specific to each patient's tumor were coadministered subcutaneously with the adjuvant poly-ICLC on a prime-boost schedule [74.•]. Robust ex vivo $\mathrm{T}$ cell responses as measured by IFN- $\gamma$ ELISPOT were detected in all six patients. Sixty percent of immunizing epitopes induced CD4+ $\mathrm{T}$ cell responses (including 18\% ex vivo), whereas $16 \%$ of immunizing epitopes induced CD8 responses. Complete regression of all metastatic melanoma and a broadening of the $T$ cell repertoire was seen in two patients who had melanoma recurrence after vaccination and subsequently were treated with the PD-1 inhibitor pembrolizumab; the four patients with stage III melanoma remained without melanoma recurrence after 32 months from vaccination start. Another clinical trial using an mRNA vaccine called IVAC MUTANOME was conducted in 13 patients with melanoma [75]. Robust CD4+ T cell responses against $60 \%$ of vaccine epitopes and $\mathrm{CD} 8+\mathrm{T}$ cell responses against $25 \%$ of vaccine epitopes were detected; in a subset of two patients, vaccine-induced $\mathrm{T}$ cell processing, including tumor mutation calling, and prediction of peptide binding affinity for HLA class I and other epitope selection criteria. c Vaccine manufacturing: validated epitopes are selected for incorporation into the personalized cancer vaccine, which is administered to patients in combination with an immune adjuvant. After final formulation and quality control, the peptide vaccine is administered to the patient and immune responses are monitored

infiltration and neoantigen-specific $\mathrm{T}$ cell mediated killing of autologous melanoma cells were observed. There was also a preliminary signal of antitumor activity: all eight patients who had no radiographic evidence of metastasis remained tumor-free during the entire study follow-up. Moreover, two of five patients with metastatic disease at baseline had objective responses following the vaccine alone, and one patient who was discontinued from vaccination on the trial because of rapid progression had a complete response after receiving a PD-1 inhibitor. All three studies demonstrated that neoantigen-based vaccination is feasible, safe, and importantly, induced robust $\mathrm{T}$ cell responses in all vaccinated patients. Furthermore, they suggest that personalized vaccination against neoantigens can be synergistic in combination with subsequently administered ICI, providing a strong rationale for combining these vaccines with ICI. Clinical trials assessing a neoantigen long-peptide vaccine in combination with nivolumab (NCT02897765) and a personalized mRNA mutanome vaccine in combination with atezolizumab (NCT03289962) are ongoing. Beyond the fact that neoantigen are promising targets for personalized vaccines, there is no agreement on the best formulation, adjuvants, and delivery to be used.

\section{Conclusion}

To increase the number of patients who will derive benefit from PD-1/PD-L1 inhibition, combined approaches are necessary. As reviewed here, pharmacologically modified cytokine therapy and a novel approach to cancer vaccines targeting tumor neoantigens, enabled by vastly improved sequencing technology and computational capabilities, may turn these two "old" immunotherapy approaches into powerful tools for effective combination immunotherapy. 
Funding Information PAO receives consulting fees from: Bristol-Myers Squibb, Merck, Genentech, Celldex, CytomX, Alexion, Novartis, Pfizer, Amgen, and research funding from Bristol-Myers Squibb, Genentech, Merck, AZ/MedImmune, CytomX, Celldex, ArmoBiosciences.

\section{Compliance with Ethical Standards}

Conflict of Interest Romualdo Barroso-Sousa declares that he has no conflict of interest.

Patrick A. Ott has received research funding from Bristol-Myers Squibb, Genentech, Merck, AstraZeneca/MedImmune, CytomX Therapeutics, Celldex, and Armo Biosciences; and has received compensation from Bristol-Myers Squibb, Merck, Genentech, Celldex, CytomX Therapeutics, Pfizer, Alexion, Amgen, and Novartis for service as a consultant.

Human and Animal Rights and Informed Consent This article does not contain any studies with human or animal subjects performed by any of the authors.

\section{References}

Papers of particular interest, published recently, have been highlighted as:

- Of importance

-• Of major importance

1. Brahmer JR, Tykodi SS, Chow LQ, et al. Safety and activity of antiPD-L1 antibody in patients with advanced cancer. N Engl J Med. 2012;366(26):2455-65.

2. Topalian SL, Hodi FS, Brahmer JR, Gettinger SN, Smith DC, McDermott DF, et al. Safety, activity, and immune correlates of anti-PD-1 antibody in cancer. N Engl J Med. 2012;366(26):244354.

3. Topalian SL, Drake CG, Pardoll DM. Immune checkpoint blockade: a common denominator approach to cancer therapy. Cancer Cell. 2015;27(4):450-61.

4. Larkin J, Chiarion-Sileni V, Gonzalez R, Grob JJ, Cowey CL, Lao $\mathrm{CD}$, et al. Combined Nivolumab and Ipilimumab or monotherapy in untreated melanoma. N Engl J Med. 2015;373(1):23-34.

5. Wolchok JD, Chiarion-Sileni V, Gonzalez R, Rutkowski P, Grob JJ, Cowey CL, et al. Overall survival with combined Nivolumab and Ipilimumab in advanced melanoma. N Engl J Med. 2017;377(14): 1345-56.

6. Chen DS, Mellman I. Oncology meets immunology: the cancerimmunity cycle. Immunity. 2013;39(1):1-10.

7. Rosenberg SA. IL-2: the first effective immunotherapy for human cancer. J Immunol (Baltimore, Md : 1950). 2014;192(12):5451-8.

8. Wang X, Rickert M, Garcia KC. Structure of the quaternary complex of interleukin-2 with its alpha, beta, and gammac receptors. Science. 2005;310(5751):1159-63.

9. Wrangle JM, Patterson A, Johnson CB, Neitzke DJ, Mehrotra S, Denlinger CE, et al. IL-2 and beyond in Cancer immunotherapy. J Interf Cytokine Res. 2018;38(2):45-68.

10. Boyman O, Sprent J. The role of interleukin-2 during homeostasis and activation of the immune system. Nat Rev Immunol. 2012;12(3):180-90.

11. Lafreniere R, Rosenberg SA. Successful immunotherapy of murine experimental hepatic metastases with lymphokine-activated killer cells and recombinant interleukin 2. Cancer Res. 1985;45(8): 3735-41.
12. Fyfe G, Fisher RI, Rosenberg SA, Sznol M, Parkinson DR, Louie AC. Results of treatment of 255 patients with metastatic renal cell carcinoma who received high-dose recombinant interleukin- 2 therapy. J Clin Oncol. 1995;13(3):688-96.

13. Klapper JA, Downey SG, Smith FO, et al. High-dose interleukin-2 for the treatment of metastatic renal cell carcinoma : a retrospective analysis of response and survival in patients treated in the surgery branch at the National Cancer Institute between 1986 and 2006. Cancer. 2008;113(2):293-301.

14. Rosenberg SA, Yang JC, Topalian SL, et al. Treatment of 283 consecutive patients with metastatic melanoma or renal cell cancer using high-dose bolus interleukin 2. JAMA. 1994;271(12):907-13.

15. Atkins MB, Lotze MT, Dutcher JP, Fisher RI, Weiss G, Margolin K, et al. High-dose recombinant interleukin 2 therapy for patients with metastatic melanoma: analysis of 270 patients treated between 1985 and 1993. J Clin Oncol. 1999;17(7):2105-16.

16. Atkins MB, Kunkel L, Sznol M, Rosenberg SA. High-dose recombinant interleukin-2 therapy in patients with metastatic melanoma: long-term survival update. Cancer J Sci Am. 2000;6(Suppl 1):S114.

17. Alva A, Daniels GA, Wong MK, et al. Contemporary experience with high-dose interleukin-2 therapy and impact on survival in patients with metastatic melanoma and metastatic renal cell carcinoma. Cancer Immunol Immunother: CII. 2016;65(12):1533-44.

18. Milla P, Dosio F, Cattel L. PEGylation of proteins and liposomes: a powerful and flexible strategy to improve the drug delivery. Curr Drug Metab. 2012;13(1):105-19.

19. Chantale Bernatchez CH, Tannir NM, Kluger H, Tetzlaff M, Bentebibel SE, Jackson N, Gergel I, Tagliaferri M, Zalevsky J, Hoch U, Imperiale M, Aung S, Hwu P, Sznol M, Hurwitz M, Diab A (2016) A CD122-biased agonist increases CD8+ T cells and natural killer cells in the tumor microenvironment; making cold tumors hot with NKTR-214 31st Annual Meeting and Associated Programs of the Society for Immunotherapy of Cancer (SITC 2016)

20. Brunet JF, Denizot F, Luciani MF, et al. A new member of the immunoglobulin superfamily-CTLA-4. Nature. 1987;328(6127): 267-70.

21. Krummel MF, Allison JP. CD28 and CTLA-4 have opposing effects on the response of $\mathrm{T}$ cells to stimulation. J Exp Med. 1995;182(2):459-65.

22. Leach DR, Krummel MF, Allison JP. Enhancement of antitumor immunity by CTLA-4 blockade. Science. 1996;271(5256):1734-6.

23. Ishida Y, Agata Y, Shibahara K, Honjo T. Induced expression of PD-1, a novel member of the immunoglobulin gene superfamily, upon programmed cell death. EMBO J. 1992;11(11):3887-95.

24. Freeman GJ, Long AJ, Iwai Y, Bourque K, Chernova T, Nishimura $\mathrm{H}$, et al. Engagement of the PD-1 immunoinhibitory receptor by a novel B7 family member leads to negative regulation of lymphocyte activation. J Exp Med. 2000;192(7):1027-34.

25. West EE, Jin HT, Rasheed AU, Penaloza-MacMaster P, Ha SJ, Tan WG, et al. PD-L1 blockade synergizes with IL-2 therapy in reinvigorating exhausted T cells. J Clin Invest. 2013;123(6):2604 15

26. AdiDiab NT, Cho D, Papadimitrakopoulou V, Bernatchez C, Haymaker C, Bentebibel SE, Curti B, Wong M, Tykodi S, Puzanov I, Smalberg I, Gergel I, Tagliaferri M, Zalevsky J, Hoch U, Aung S, Imperiale M, Clemens W, Kluger H, Hurwitz M, Hwu P, Sznol M (2017) Preliminary safety, efficacy and biomarker results from the Phase 1/2 study of CD-122-biased agonist NKTR214 plus nivolumab in patients with locally advanced/metastatic solid tumors. 32nd Annual Meeting and Pre-Conference Programs of the Society for Immunotherapy of Cancer (SITC 2017).This study shows that the combination of NKTR-214 with the PD-1 inhibitor nivolumab is safely administered, With promissing clinical activity in patients with advanced cancers. 
27. Munger W, DeJoy SQ, Jeyaseelan R Sr, et al. Studies evaluating the antitumor activity and toxicity of interleukin-15, a new $\mathrm{T}$ cell growth factor: comparison with interleukin-2. Cell Immunol. 1995;165(2):289-93.

28. Rubinstein MP, Kadima AN, Salem ML, Nguyen CL, Gillanders WE, Cole DJ. Systemic administration of IL-15 augments the antigen-specific primary $\mathrm{CD} 8+\mathrm{T}$ cell response following vaccination with peptide-pulsed dendritic cells. J Immunol (Baltimore, Md : 1950). 2002;169(9):4928-35.

29. Liu RB, Engels B, Schreiber K, et al. IL-15 in tumor microenvironment causes rejection of large established tumors by $\mathrm{T}$ cells in a noncognate $\mathrm{T}$ cell receptor-dependent manner. Proc Natl Acad Sci U S A. 2013;110(20):8158-63.

30. Conlon KC, Lugli E, Welles HC, et al. Redistribution, hyperproliferation, activation of natural killer cells and CD8 T cells, and cytokine production during first-in-human clinical trial of recombinant human interleukin-15 in patients with cancer. J Clin Oncol. 2015;33(1):74-82.

31. Bergamaschi C, Rosati M, Jalah R, Valentin A, Kulkarni V, Alicea $\mathrm{C}$, et al. Intracellular interaction of interleukin-15 with its receptor alpha during production leads to mutual stabilization and increased bioactivity. The. J Biol Chem. 2008;283(7):4189-99.

32. Rubinstein MP, Kovar M, Purton JF, et al. Converting IL-15 to a superagonist by binding to soluble IL-15R \{alpha\}. Proc Natl Acad Sci U S A. 2006;103(24):9166-71.

33. Stoklasek TA, Schluns KS, Lefrancois L. Combined IL-15/IL15Ralpha immunotherapy maximizes IL-15 activity in vivo. J Immunol (Baltimore, Md : 1950). 2006;177(9):6072-80.

34. Mortier E, Quemener A, Vusio P, et al. Soluble interleukin-15 receptor alpha (IL-15R alpha)-sushi as a selective and potent agonist of IL-15 action through IL-15R beta/gamma. Hyperagonist IL-15 x IL-15R alpha fusion proteins. J Biol Chem. 2006;281(3):1612-9.

35. Dubois S, Patel HJ, Zhang M, Waldmann TA, Muller JR. Preassociation of IL-15 with IL-15R alpha-IgG1-Fc enhances its activity on proliferation of $\mathrm{NK}$ and $\mathrm{CD} 8+/ \mathrm{CD} 44$ high T cells and its antitumor action. J Immunol (Baltimore, Md : 1950). 2008;180(4): 2099-106.

36. Rhode PR, Egan JO, Xu W, Hong H, Webb GM, Chen X, et al. Comparison of the Superagonist complex, ALT-803, to IL15 as Cancer Immunotherapeutics in animal models. Cancer Immunol Res. 2016;4(1):49-60.

37. Wrangle JM, Velcheti V, Patel MR, et al. ALT-803, an IL-15 superagonist, in combination with nivolumab in patients with metastatic non-small cell lung cancer: a non-randomised, open-label, phase $1 \mathrm{~b}$ trial. Lancet Oncol. 2018;19(5):694-704 This study shows that the combination of the IL-15 superagonist ALT803 with the PD-1 inhibitor nivolumab is safely administered in an outpatient setting, with promissing clinical activity in patients with refractory NSCLC.

38. Kirkwood JM, Strawderman MH, Ernstoff MS, Smith TJ, Borden EC, Blum RH. Interferon alfa-2b adjuvant therapy of high-risk resected cutaneous melanoma: the eastern cooperative oncology group trial EST 1684. J Clin Oncol. 1996;14(1):7-17.

39. Kirkwood JM, Ibrahim JG, Sosman JA, et al. High-dose interferon alfa- $2 \mathrm{~b}$ significantly prolongs relapse-free and overall survival compared with the GM2-KLH/QS-21 vaccine in patients with resected stage IIB-III melanoma: results of intergroup trial E1694/S9512/ C509801. J Clin Oncol. 2001;19(9):2370-80.

40. Mocellin S, Pasquali S, Rossi CR, Nitti D. Interferon alpha adjuvant therapy in patients with high-risk melanoma: a systematic review and meta-analysis. J Natl Cancer Inst. 2010;102(7):493-501.

41. Weber J, Mandala M, Del Vecchio M, et al. Adjuvant Nivolumab versus Ipilimumab in resected stage III or IV melanoma. N Engl J Med. 2017;377(19):1824-35.
42. Eggermont AMM, Blank CU, Mandala M, Long GV. Adjuvant Pembrolizumab versus Placebo in Resected Stage III Melanoma. N Engl J Med. 2018;378(19):1789-801.

43. Choueiri TK, Motzer RJ. Systemic therapy for metastatic renal-cell carcinoma. N Engl J Med. 2017;376(4):354-66.

44. Terawaki S, Chikuma S, Shibayama S, Hayashi T, Yoshida T, Okazaki T, et al. IFN-alpha directly promotes programmed cell death-1 transcription and limits the duration of T cell-mediated immunity. J Immunol (Baltimore, Md : 1950). 2011;186(5):27729.

45. Atkins MB, Hodi FS, Thompson JA, et al. Pembrolizumab plus Pegylated interferon alfa- $2 \mathrm{~b}$ or Ipilimumab for advanced melanoma or renal cell carcinoma: dose-finding results from the phase $\mathrm{Ib}$ KEYNOTE-029 study. Clin Cancer Res. 2018;24:1805-15.

46. Asadullah K, Sterry W, Volk HD. Interleukin-10 therapy-review of a new approach. Pharmacol Rev. 2003;55(2):241-69.

47. Fujii S, Shimizu K, Shimizu T, Lotze MT. Interleukin-10 promotes the maintenance of antitumor CD8(+) T-cell effector function in situ. Blood. 2001;98(7):2143-51.

48. Emmerich J, Mumm JB, Chan IH, et al. IL-10 directly activates and expands tumor-resident CD8(+) T cells without de novo infiltration from secondary lymphoid organs. Cancer Res. 2012;72(14):3570 81 .

49. Chan IH, Wu V, Bilardello M, Mar E, Oft M, van Vlasselaer P, et al. The potentiation of IFN-gamma and induction of cytotoxic proteins by Pegylated IL-10 in human CD8 T cells. J Interf Cytokine Res. 2015;35(12):948-55.

50. Naing A, Papadopoulos KP, Autio KA, Ott PA, Patel MR, Wong DJ, et al. Safety, antitumor activity, and immune activation of Pegylated recombinant human Interleukin-10 (AM0010) in patients with advanced solid tumors. J Clin Oncol. 2016;34(29):3562-9.

51.• Aung Naing DJW, Infante JR, Papadopoulos K, Aljumaily R, Korn WM, Schneider JG, Patel M, Autio KA, Falckook GS, Gabrail NY, Rojo B, Ratti N, McCauley S, Hung A, Van Vlasselaer P, Brown GL, Garon EB, Tannir NM, Oft M (2017) Immune and tumor responses to human IL-10 (AM0010, Pegilodecakin) alone or in combination with immune checkpoint blockade. 32nd Annual Meeting and Pre-Conference Programs of the Society for Immunotherapy of Cancer (SITC 2017). This study shows that the combination of the PEGylated IL-10 with PD-1 inhibitors is safely administered, With promissing clinical activity in patients with advanced cancers.

52. $\mathrm{Hu} \mathrm{Z}, \mathrm{Ott} \mathrm{PA}, \mathrm{Wu}$ CJ. Towards personalized, tumour-specific, therapeutic vaccines for cancer. Nat Rev Immunol. 2018;18(3):168-82.

53. Brown SD, Warren RL, Gibb EA, Martin SD, Spinelli JJ, Nelson $\mathrm{BH}$, et al. Neo-antigens predicted by tumor genome meta-analysis correlate with increased patient survival. Genome Res. 2014;24(5): $743-50$.

54. Rooney MS, Shukla SA, Wu CJ, Getz G, Hacohen N. Molecular and genetic properties of tumors associated with local immune cytolytic activity. Cell. 2015;160(1-2):48-61.

55. Giannakis M, Mu XJ, Shukla SA, Qian ZR, Cohen O, Nishihara R, et al. Genomic Correlates of Immune-Cell Infiltrates in Colorectal Carcinoma. Cell Rep. 2016;17(4):1206.

56. Howitt BE, Shukla SA, Sholl LM, Ritterhouse LL, Watkins JC, Rodig S, et al. Association of Polymerase e-mutated and microsatellite-instable endometrial cancers with Neoantigen load, number of tumor-infiltrating lymphocytes, and expression of PD-1 and PD-L1. JAMA Oncol. 2015;1(9):1319-23.

57. Snyder A, Makarov V, Merghoub T, Yuan J, Zaretsky JM, Desrichard A, et al. Genetic basis for clinical response to CTLA4 blockade in melanoma. N Engl J Med. 2014;371:2189-99.

58. Rizvi NA, Hellmann MD, Snyder A, et al. Cancer immunology Mutational landscape determines sensitivity to PD-1 blockade in non-small cell lung cancer. Science. 2015;348(6230):124-8. 
59. Le DT, Uram JN, Wang H, et al. PD-1 blockade in tumors with mismatch-repair deficiency. N Engl J Med. 2015;372(26):2509-20.

60. Le DT, Durham JN, Smith KN, et al. Mismatch repair deficiency predicts response of solid tumors to PD-1 blockade. Science. 2017;357(6349):409-13.

61. van Rooij N, van Buuren MM, Philips D, Velds A, Toebes M, Heemskerk B, et al. Tumor exome analysis reveals neoantigenspecific T-cell reactivity in an ipilimumab-responsive melanoma. J Clin Oncol. 2013;31(32):e439-42.

62. Matsushita H, Vesely MD, Koboldt DC, Rickert CG, Uppaluri R, Magrini VJ, et al. Cancer exome analysis reveals a T-cell-dependent mechanism of cancer immunoediting. Nature. 2012;482(7385):400-4.

63. DuPage M, Mazumdar C, Schmidt LM, Cheung AF, Jacks T. Expression of tumour-specific antigens underlies cancer immunoediting. Nature. 2012;482(7385):405-9.

64. Castle JC, Kreiter S, Diekmann J, Löwer M, van de Roemer N, de Graaf J, et al. Exploiting the mutanome for tumor vaccination. Cancer Res. 2012;72(5):1081-91.

65. Yadav M, Jhunjhunwala S, Phung QT, Lupardus P, Tanguay J, Bumbaca $\mathrm{S}$, et al. Predicting immunogenic tumour mutations by combining mass spectrometry and exome sequencing. Nature. 2014;515(7528):572-6.

66. Tran E, Turcotte S, Gros A, Robbins PF, Lu YC, Dudley ME, et al. Cancer immunotherapy based on mutation-specific CD4+ T cells in a patient with epithelial cancer. Science. 2014;344(6184):641-5.

67. Gubin MM, Zhang X, Schuster H, Caron E, Ward JP, Noguchi T, et al. Checkpoint blockade cancer immunotherapy targets tumourspecific mutant antigens. Nature. 2014;515(7528):577-81.

68. Kreiter S, Vormehr M, van de Roemer N, Diken M, Löwer M, Diekmann J, et al. Mutant MHC class II epitopes drive therapeutic immune responses to cancer. Nature. 2015;520(7549):692-6.

69. Tran E, Robbins PF, Lu YC, Prickett TD, Gartner JJ, Jia L, et al. Tcell transfer therapy targeting mutant KRAS in Cancer. N Engl J Med. 2016;375(23):2255-62.
70. Kantoff PW, Higano CS, Shore ND, Berger ER, Small EJ, Penson DF, et al. Sipuleucel-T immunotherapy for castration-resistant prostate cancer. N Engl J Med. 2010;363(5):411-22.

71. Kuai R, Ochyl LJ, Bahjat KS, Schwendeman A, Moon JJ. Designer vaccine nanodiscs for personalized cancer immunotherapy. Nat Mater. 2017;16(4):489-96.

72.• Schreiber RD, Old LJ, Smyth MJ. Cancer immunoediting: integrating immunity's roles in cancer suppression and promotion. Science. 2011;331(6024):1565-70. References 72-74 are the first clinical studies showing that neoantigen-based anticancer vaccines are safely administered, the manufacturing is timely feasible and induce durable neoantigen-specific $T$ cell responses in patients with locally advanced and metastatic melanoma.

73.• Carreno BM, Magrini V, Becker-Hapak M, Kaabinejadian S, Hundal J, Petti AA, et al. Cancer immunotherapy A dendritic cell vaccine increases the breadth and diversity of melanoma neoantigen-specific T cells. Science. 2015;348(6236):803-8. References $\mathbf{7 2 - 7 4}$ are the first clinical studies showing that neoantigen-based anticancer vaccines are safely administered, the manufacturing is timely feasible and induce durable neoantigen-specific $T$ cell responses in patients with locally advanced and metastatic melanoma.

74.• Ott PA, Hu Z, Keskin DB, et al. An immunogenic personal neoantigen vaccine for patients with melanoma. Nature. 2017;547(7662):217-21 References 72-74 are the first clinical studies showing that neoantigen-based anticancer vaccines are safely administered, the manufacturing is timely feasible and induce durable neoantigen-specific $T$ cell responses in patients with locally advanced and metastatic melanoma.

75. Sahin U, Derhovanessian E, Miller M, Kloke BP, Simon P, Löwer $\mathrm{M}$, et al. Personalized RNA mutanome vaccines mobilize polyspecific therapeutic immunity against cancer. Nature. 2017;547(7662):222-6. 